OPEN ACCESS

Edited by:

Dalin Tang,

Worcester Polytechnic Institute,

United States

Reviewed by:

Minliang Liu,

Georgia Institute of Technology,

United States

Liang Wang,

Southeast University, China

*Correspondence:

Xueying Huang

xhuang@xmu.edu.cn

tThese authors have contributed equally to this work

Specialty section:

This article was submitted to Computational Physiology and Medicine,

a section of the journal

Frontiers in Physiology

Received: 31 May 2021 Accepted: 28 June 2021

Published: 21 July 2021

Citation:

Deng L, Zuo H, Li A, Yang C and Huang $X$ (2021) Numerical Simulation

Study on the Mechanism

of Formation of Apical Aneurysm in Hypertrophic Cardiomyopathy With

Midventricular Obstruction.

Front. Physiol. 12:717717.

doi: 10.3389/fphys.2021.717717

\section{Numerical Simulation Study on the Mechanism of Formation of Apical Aneurysm in Hypertrophic Cardiomyopathy With Midventricular Obstruction}

\author{
Long Deng ${ }^{1 \dagger}$, Heng Zuo ${ }^{2 t}$, An $\mathrm{Li}^{3}$, Chun Yang ${ }^{4}$ and Xueying Huang ${ }^{3,5 *}$ \\ ${ }^{1}$ Department of Cardiac Surgery, Fuwai Hospital, Chinese Academy of Medical Sciences, Beijing, China, ${ }^{2}$ School \\ of Mathematical Sciences, Sichuan Normal University, Chengdu, China, ${ }^{3}$ School of Mathematical Sciences, Xiamen \\ University, Xiamen, China, ${ }^{4}$ Network Technology Research Institute, China United Network Communications Co., Ltd., \\ Beijing, China, ${ }^{5}$ Department of Mathematics, Worcester Polytechnic Institute, Worcester, MA, United States
}

Apical aneurysm was observed to be associated with midventricular obstruction (MVO) in hypertrophic cardiomyopathy ( $\mathrm{HCM})$. To investigate the genesis of the apical aneurysm, the idealized numerical left ventricular models (finite-element left ventricle models) of the healthy left ventricle, subaortic obstruction, and midventricular obstruction in HCM of left ventricle were created. The mechanical effects in the formation of apical aneurysm were determined by comparing the myofiber stress on the apical wall between these three models (healthy, subaortic obstruction, and midventricular obstruction models). In comparing the subaortic obstruction model and MVO model with $\mathrm{HCM}$, it was found that, at the time of maximum pressure, the maximum value of myofiber stress in MVO model was 75.0\% higher than that in the subaortic obstruction model $(654.5 \mathrm{kPa}$ vs. $373.9 \mathrm{kPa})$. The maximum stress on the apex of LV increased $79.9,69.3,117.8 \%$ than that on the myocardium around the apex in healthy model, subaortic obstruction model, and MVO model, respectively. Our results indicated that high myofiber stress on the apical wall might initiate the formation process of the apical aneurysm.

Keywords: hypertrophic cardiomyopathy, midventricular obstruction, stress, finite element model, strain, left ventricle

\section{INTRODUCTION}

Hypertrophic cardiomyopathy (HCM) is an inherited myocardial disease defined by unexplained cardiac hypertrophy, which occurs in about 1 of every 500 adults in the general population (Semsarian et al., 2015). The manifestation of HCM disease is mostly dependent on the hypertrophy and/or obstruction at different intracavitary position (Efthimiadis et al., 2013). Midvertricular obstruction (MVO) is the impedance to flow at the middle level of the left ventricle, while subaortic 
obstruction is caused by hypertrophy in the basal septum (Falicov et al., 1976). It's been observed that the presence of MVO is more likely to relate with the formation of apical aneurysm (Minami et al., 2011), which do not happen in HCM patients with mere subaortic obstruction. Although HCM with subaortic obstruction has been adequately studied to date, the clinical features of midventricular obstruction and the mechanisms for the formation of apical aneurysm remain undetermined (Harada et al., 2001).

It has been well accepted that the increase in ventricular wall stress will lead to various pathophysiological process in the heart, including hypertrophy, ischemia and fibrosis (Grossman, 1980; Blaauw et al., 2010; Genet et al., 2014; You et al., 2018). Considering the pathological basis of apical aneurysm is myocardial fibrosis, we hypothesized that the increased ventricular wall stress might contribute to the formation of apical aneurysm in MVO. However, the information of the wall stress was lacking with current clinical examinations. A computational ventricular model of the heart that use finite element method may provide the information of ventricular wall stress and strain, which has been adopted to analyze cardiac computational mechanics and becomes increasingly common (Walker et al., 2005; Tang et al., 2016; Yu et al., 2019; Yin et al., 2020). The present study was undertaken to investigate the mechanisms of the formation of apical aneurysm in HCM, based on the numerical simulation results of three constructed idealized finite element models with different positions of myocardial hypertrophy (healthy, subaortic obstruction, and midventricular obstruction). The obtained systolic myocardial stress/strain in these models were compared to determine the mechanical effects of different hypertrophy positions.

\section{MATERIALS AND METHODS}

\section{Idealized Geometries}

Three idealized geometries of left ventricle with no obstruction, subaortic obstruction, and midventricular obstruction of hypertrophic cardiomyopathy (Figure 1) were constructed. The geometry of Model 2 and Model 3 were adjusted based on the ellipsoid model (Model 1), which is the idealized model of healthy left ventricle. The mathematical models for inner/outer surfaces (endocardium/epicardium) of Model 1 were given by,

$$
\left\{\begin{array}{l}
x=R \sin \theta \cos \varphi \\
y=R \sin \theta \sin \varphi \\
z=\mathrm{R}-\cos \pi
\end{array}\right.
$$

where $0 \quad 2,0 \quad f / 2 . \mathrm{R} 2 \mathrm{~cm}$ represents the endocardium model; R 3 represents the epicardium model. The positions of myocardial hypertrophy were numerically adjusted (Figure 1). Model 2 is the model of subaortic (basal septum) hypertrophy, and Model 3 is the MVO model in which the hypertrophy occurs at the midcavitary level. The figure of the slice, on which the hypertrophy was most severe, for Model 2 and 3 was presented on Figure 2.

\section{Finite Element Modeling}

\section{Solid Model}

The material of LV was assumed to be hyperelastic, isotropic, nearly incompressible, and homogeneous. The governing equations for the structure models are (summation convention is used) given as follows,

$$
\begin{gathered}
\rho v_{i, t t}=\sigma_{i j, j}, i, j=1,2,3 \text { (Equation of Motion) } \\
\varepsilon_{i j}=\left(\mathrm{v}_{i, j}+\mathrm{v}_{j, i}+\mathrm{v}_{\alpha, i} \mathrm{v}_{\alpha, j}\right) / 2, i, j, \alpha=1,2,3,
\end{gathered}
$$

where $t$ stands for time, $i, j$ and label spatial coordinates, $\mathbf{v}$ is the solid displacement vector, $\sigma=\left[\sigma_{i, j}\right]$ is the stress tensor, and $\rho$ is material density. $f_{\text {., }}$ stands for derivative with respect to the $j$ th variable. Here, body force due to gravity was ignored. The nonlinear Mooney-Rivlin model was used to describe the material properties of the LV material with parameter values chosen to match experimental data available (Sacks and Chuong, 1993; Bathe, 2002; Humphrey, 2002; Tang et al., 2010). The strain energy function for the modified MooneyRivlin model is given by Bathe (2002) andTang et al. (2010),

$$
W=c_{1}\left(I_{1}-3\right)+c_{2}\left(I_{2}-3\right)+D_{1}\left[\exp \left(D_{2}\left(I_{1}-3\right)\right)-1\right]
$$

where $I_{1}=\sum C_{\mathrm{ii}}$, and $\mathrm{I}_{2}=\frac{1}{2}\left(I_{1}^{2}-C_{\mathrm{ij}} C_{\mathrm{ij}}\right)$, are the first and second strain invariants, $\mathbf{C}=\left[C_{\mathrm{ij}}\right]=\mathbf{X}^{T} \mathbf{X}$ is the right Cauchy-Green deformation tensor, $\mathbf{X}=\left[X_{\mathrm{ij}}\right]=\left[\partial x_{i} / \partial a_{j}\right]$, where $x_{i}$ is the current position, $a_{i}$ is the original position, $n_{f}$ is the fiber direction, and $c_{i}$, and $D_{i}$ are material constants chosen to match experimental measurements: $c_{1}=18.4$ $\mathrm{kPa}, c_{2}=0, D_{1}=7.2 \mathrm{kPa}$, and $D_{2}=2.0$ (Bathe, 2002; Yang et al., 2007).

\section{Boundary Conditions}

The apex of LV was constrained from moving in the circumferential direction, and the top of the LV is fixed. The pressure condition applied here was obtained from our previous patient-specific model (Deng et al., 2018). The pulsating pressure conditions were uniformly imposed at LV endocardium wall. The maximum value of pressure (LV end of diastolic pressure) was prescribed to be $10 \mathrm{mmHg}$ and the minimum value (LV end of systolic pressure) was prescribed to be $180 \mathrm{mmHg}$ in all models.

\section{Active Model}

It's been verified that the results of wall stress in active models were significant different with those obtained from passive model (Huang et al., 2021). To be realistic, the active model was employed in this study for more accurate simulation of cardiac biomechanics. Since the models were idealized model, the simulation started from the zero-load state. After the inlet pressure increased, the LV expanded in both circumferential axis and longitudinal axis directions. In our model, the active tension was implemented by specifying the external forces on epicardium during isovolumic systole phase. The external forces values were obtained by scaling the internal pressure of LV to match the ideal LV volumetric changing trending during one cardiac cycle, which 
A Model 1

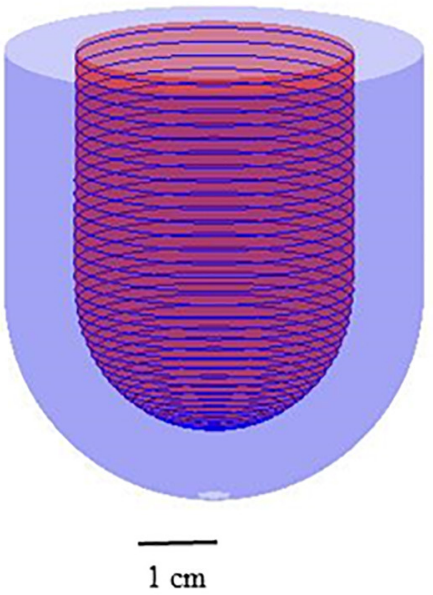

B Model 2

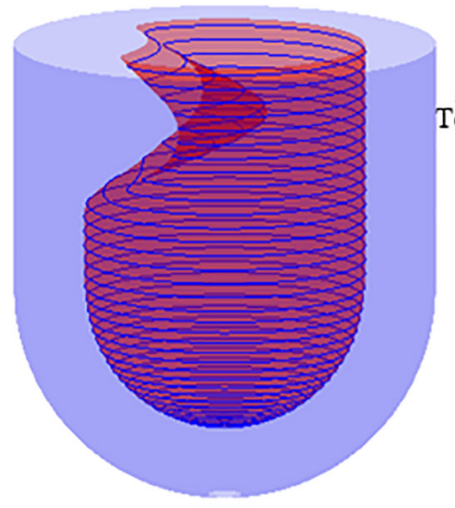

C Model 3

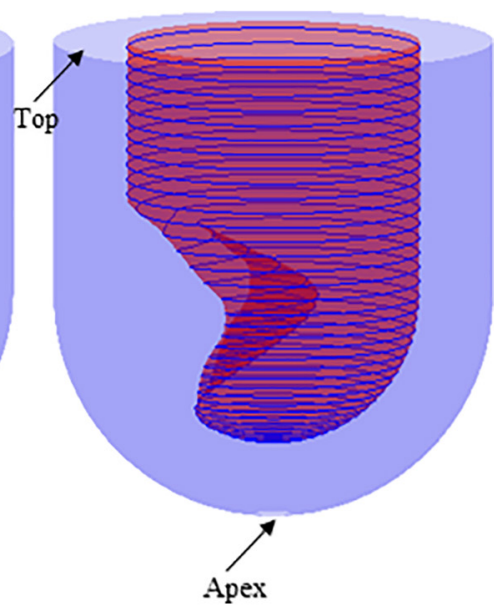

FIGURE 1 | 3D Geometries for the construction of three idealized models. (A) Model 1: healthy model of left ventricle; (B) Model 2: subaortic obstruction of left ventricle in hypertrophic cardiomyopathy; (C) Model 3 (MVO model): midventricular obstruction of left ventricle in hypertrophic cardiomyopathy.

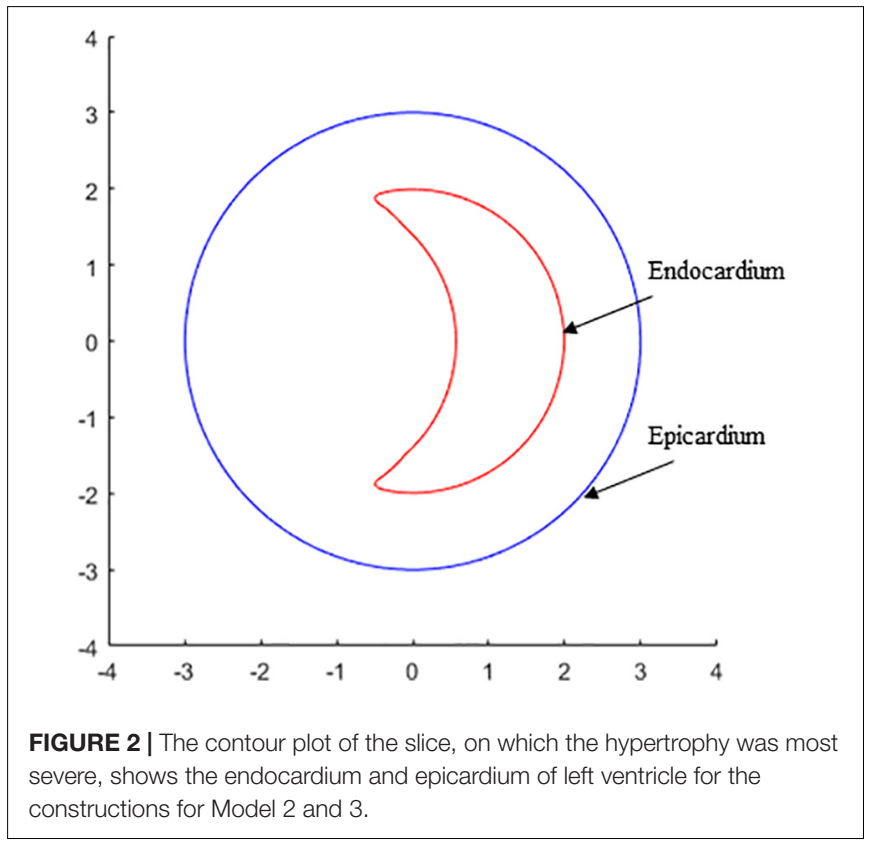

has been verified by clinical measured data (Deng et al., 2018). The real LV active contraction motion was implemented.

\section{Mesh Generation and Solution Method}

3D tetrahedral (4-node) isoparametric displacement-based finite elements were employed. The finite element model was solved by ADINA (ADINA R\&D, Watertown, MA) using unstructured finite elements and the Newton-Raphson iteration method. Mesh analysis was performed for each model by reducing the mesh density in each dimension by $10 \%$ until differences between solutions from two consecutive meshes were negligible (less than $1 \%$ in $\mathrm{L}_{2}$-norm). More details can

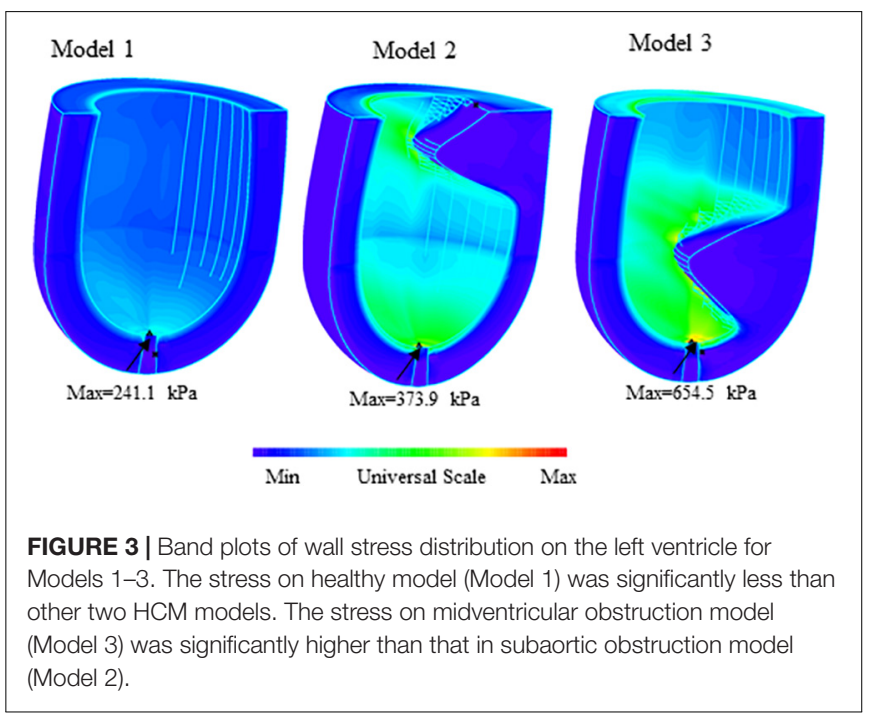

be found in previously published papers (Yang et al., 2007; Tang et al., 2011).

\section{Data Extraction and Statistical Analysis}

The stress and strain are all tensors. Therefore, the maximum principal stress (Stress- $\mathrm{P}_{1}$ ) and maximum principal strain (Strain- $\mathrm{P}_{1}$ ) at each node were chosen as wall stress and wall strain, respectively, for convenience. The data for wall stress and wall strain of all integral nodes on the inner boundary of left ventricle (Endocardium) were extracted from the simulation results. The nodes on the region of apex and around apex of LV were selected for investigation. The data was expressed as mean \pm SD. Paired $t$-test was used to compare the differences of wall stress/strain between different models. $P<0.05$ was established as the level of statistical 
TABLE 1 | Summary of wall stress and wall strain results in three models.

\begin{tabular}{lcccc}
\hline & Mode1 & Model 2 & Model 3 & Diff (\%) \\
\hline MaxStress $(\mathrm{kPa})$ & 241.1 & 373.9 & 654.5 & 75.0 \\
AverageStress $(\mathrm{kPa})$ & 67.5 & 127.4 & 138.9 & 9.0 \\
MaxStrain $(\mathrm{kPa})$ & 0.243 & 0.414 & 0.516 & 24.7 \\
AverageStrain $(\mathrm{kPa})$ & 0.117 & 0.230 & 0.273 & 18.9
\end{tabular}

Diff: the difference between MVO model and Subaortic Obstruction Model (base model).

significance. All statistical analysis was performed with $\mathrm{R}$ software (version 3.5.1).

\section{RESULTS}

An overview of simulation results of wall stress obtained from three models are shown in Figure 3. Table 1 summarized and compared the maximum and average values of stress and strain results on the apex of left ventricle for Models 1-3.

\section{Stress in the MVO Model Was Higher Than That in the Subaortic Obstruction Model}

Figure 4 presents the band plots of stress on the cutting position (Figure 4A) for each models. The stress distribution on the apex of LV was enlarged to present more details. It was found that the maximum stress value on the apex of LV in Model 3 (MVO model) is $75.05 \%$ higher than that in subaortic obstruction model $(654.5 \mathrm{kPa}$ vs. $373.9 \mathrm{kPa})$. The average value of stress on the apex area of LV in Model 3 is $138.9 \mathrm{kPa}$, which is slightly higher than that in Model 2 (127.4 kPa, 9\% increase). The wall stress in Model 3 on the apex of LV was statistically significantly higher than that in Model $2(\mathrm{p}=0.014)$.

\section{Stress Level on the Apex of LV Was Significantly Higher Than That on the Other Part of LV}

The comparisons of maximum stress on the apex of LV and myocardium around the apex (Figure 4) for three models were summarized on Table 2. It was found that the maximum wall stress on apex was $79.9,69.3$, and $117.8 \%$ higher than that on the region around apex for Models 1-3, respectively. In MVO model, the maximum stress on apex increased most significantly comparing to that on the region round apex (654.5 $\mathrm{kPa}$ vs. $300.5 \mathrm{kPa}$ ).

\section{Strain in MVO Model Was Slightly Higher Than That in Subaortic Obstruction Model}

The strain value on the apex of LV in MVO model (Model 3) was found slightly higher than that in subaortic obstruction model (Model 2) $(0.230=0.034$ vs. $0.273=0.090, \mathrm{p}<0.01)$. The maximum strain value on the apex in Model 3 was 0.516 , which is $24.7 \%$ higher than that in Model 2 (0.414).
A Cutting position

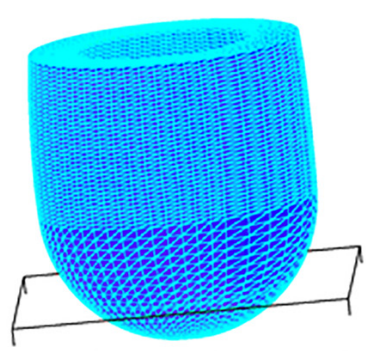

B Band plots of wall stress on the cutting position of Model 1.
C Band plots of wall stress on the cutting position of Model 3.

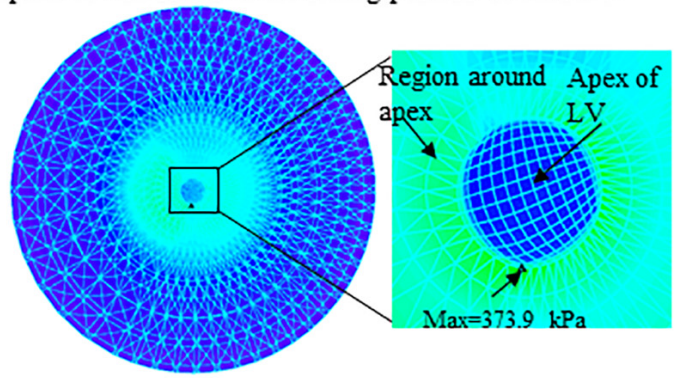

D Band plots of wall stress on the cutting position of Model 3.

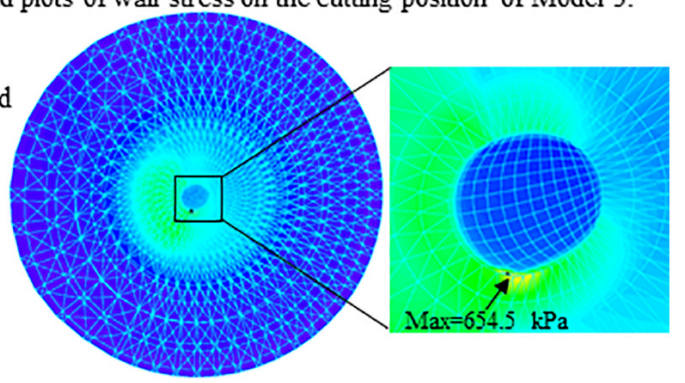

$45 \quad 150 \quad 300 \quad 500 \quad 630 \mathrm{kPa}$

FIGURE 4 | Band plots of wall stress on the apex and regions around apex of left ventricle for three models. The stress on both of the apex and region around apex in midventricular obstruction (MVO) model (Model 3) were significantly higher than that in Models 1 and 2. (A) Cutting position. (B) Band plots of wall stress on the cutting position of Model 1. (C) Band plots of wall stress on the cutting position of Model 2. (D) Band plots of wall stress on the cutting position of Model 3. 
TABLE 2 | Comparisons of maximum wall stress on apex and the region around apex of LV for Models 1-3.

\begin{tabular}{lccc}
\hline & \multicolumn{3}{c}{ Maximum Stress } \\
\cline { 2 - 4 } & Apex $\mathbf{( k P a )}$ & AroundApex $\mathbf{( k P a )}$ & Diff (\%) \\
\hline Model 1 & 241.1 & 134.0 & 79.9 \\
Model 2 & 373.9 & 220.8 & 69.3 \\
Model 3 & 654.5 & 300.5 & 117.8 \\
\hline
\end{tabular}

\section{Stress/Strain in the Healthy Model Was Significantly Less Than That in Patients' Models}

The maximum stress value on the apex for the healthy model (Model 1) was significantly less than subaortic model $(241.1 \mathrm{kPa}$ vs. $373.9 \mathrm{kPa}$ ) and MVO model (241.1 $\mathrm{kPa}$ vs. $654.5 \mathrm{kPa})$. The maximum stress value on the region around apex of LV in Model $1(134.0 \mathrm{kPa})$ is also significantly less than Model $2(220.9 \mathrm{kPa}$, $39.3 \%$ decrease) and Model $3(300.5 \mathrm{kPa}, 55.4 \%$ decrease). The maximum strain on the apex of LV was found $49.1 \%$ less than that in the subaortic obstruction model ( 0.117 vs. 0.230$)$ and $57.1 \%$ less than that in MVO model (0.117 vs. 0.273$)$.

\section{DISCUSSION}

In this study, three idealized finite element models, in which, one is the healthy model, the other two are patients' models with different positions of myocardial hypertrophy, were constructed to investigate the potential mechanical mechanisms of the formation of LV apical aneurysm in HCM patients with MVO. Our results indicated that the wall stress on the apex of LV in the MVO model was significantly higher than that in the subaortic obstruction model and healthy model. This may explain the reason that the apical aneurysm was only found in HCM patients with MVO.

Interestingly, the maximum wall stress on the apex in MVO model was $280 \mathrm{kPa}$ higher than that in subaortic obstruction model (75.0\% higher); while the average value of the wall stress on the apex in MVO model is slightly higher (9\% higher). This is because the myocardial hypertrophy in midcavitary position might affect only a small region of apex of LV. Therefore, the critical (maximum) stress was believed to be responsible for the formation of the apical aneurysm. Comparing to the healthy model, the maximum stress on the apex in subaortic obstruction model is also significantly higher $(55.1 \%)$ than that in healthy model; the maximum strain on the apex is $96.6 \%$ higher. These results suggested that the subaortic obstruction in HCM may also cause abnormal stress distributions on endocardium of LV. Future work is needed to validate these findings.

Midventricular obstruction (MVO) is a less common phenotype of HCM, it occurs in approximately $10 \%$ of entire HCM patients (Elliott et al., 2014). Compared to subaortic obstruction, patients with MVO have worse clinical prognosis, which is believed to be associated with apical aneurysm (AA) (Minami et al., 2011; Osawa et al., 2011; Efthimiadis et al., 2013).
AA could lead to ventricular arrhythmias and thromboembolic events, and eventually compromises ventricular function. In cardiovascular diseases, AA is mainly seen in patients suffering myocardial infarction, and its pathological basis is myocardial fibrosis (Friedman and Dunn, 1995). However, in HCM patients without coronary artery lesions, the mechanisms of the formation of AA remain unclear. Our results in this research indicated that, compared to subaortic obstruction, MVO has a significantly increased wall stress in the apex. The increased wall stress could cause cardiomyocytes injury, microcirculation disturbance, myocardial ischemia, and eventually myocardial fibrosis (Di Napoli et al., 2003; You et al., 2018), which might be the potential mechanisms of the formation of AA in HCM. With further validation of patient-specific simulations, we may advocate that patients with MVO should receive surgery (septal myectomy) as soon as possible to decrease the wall stress, and thereby prevent the occurrence and development of AA to avoid the associated poor prognosis.

Instead of patient-specific models, the idealized models were employed in this research. The reasons were as follows, (1) Midventricular obstruction appear alone in HCM is very rare, the patients have MVO in HCM in LV usually combined with subaortic obstruction. It's hard to obtained patient-specific data of MVO in HCM patients; (2) the geometries and hemodynamic status between different patients for MVO or subaortic obstruction patients may be quite different. The idealized models may identify the position of hypertrophy as an independent factor of the genesis of apical aneurysm.

Several improvements can be added to our models: (a) fluidstructure Interactions models (FSI) models; although the stress may be the most important factor contributes to the formation of AA, the fluid shear stress probably may contribute too. This research was the first step to investigate the mechanisms of apical aneurysm by applying finite element model method. In the future, we will apply the FSI models and investigate hemodynamic effects of the blood flow. (b) Fiber orientation and anisotropic models; the multi-layer anisotropic models may be introduced to make possible improvement in computational prediction accuracies. However, the irregular, disorganized alignment of muscle cells or myocardial disarray was found normally in the heart in HCM patients. The fiber orientation data was not available; (c) patient-specific model will be considered in the future; this is the preliminary study to demonstrate the mechanical effects of the position of hypertrophy; (d) LV remodeling approaches; Myocyte hypertrophy and myocardial fibrosis are all associated with LV remodeling (Türkoðlu et al., 2016). Therefore, it is worth adding the remodeling approached to investigate the mechanism of the formation of apical aneurysms in our future studies. (e) Follow up research should be included when patients' data are available. Although the stress in subaortic obstruction model is on the higher stress level comparing to healthy model, the AA was not found in HCM patients with subaortic obstruction. AA was only found in HCM patients with midventricular obstruction, in which the stress on the apex of LV was on the higher level comparing to subaortic obstruction model. It would be an 
interesting topic if there was a threshold of stress level for the formation of AA. The follow up research might help us better understand the mechanisms.

\section{CONCLUSION}

To investigate the mechanisms of formation of apical aneurysm in HCM, three idealized finite element models were proposed to compare the stress/strain on the apex of LV for healthy patient and patients with different position of myocardial hypertrophy (subaortic and midventricular obstruction in HCM patients). The idealized model may help us identify the position of hypertrophy as an independent factor of the genesis of apical aneurysm. It was found that the stress/strain on the apex of LV in midvertricular obstruction model was significantly higher than that on both the subaortic obstruction model and the healthy model. The obtained results suggested that the midventricular obstruction significantly increase the myofiber stress in the apex of LV, which might directly initiates the apical aneurysm. These results are preliminary studies, and the related patient-specific model studies and follow up studies in the future may help us better understand the association between midventricular obstruction and apical aneurysm.

\section{REFERENCES}

Bathe, K. (2002). Theory and Modeling Guide. Report ARD 12-10. Watertown, MA: Adina R\&D Inc.

Blaauw, E., Van Nieuwenhoven, F. A., Willemsen, P., Delhaas, T., Prinzen, F. W., Snoeckx, L. H., et al. (2010). Stretch-induced hypertrophy of isolated adult rabbit cardiomyocytes. Am. J. Physiol. Heart Circ. Physiol. 299, H780-H787.

Deng, L., Huang, X., Yang, C., Lyu, B., Duan, F., Tang, D., et al. (2018). Numerical simulation study on systolic anterior motion of the mitral valve in hypertrophic obstructive cardiomyopathy. Int. J. Cardiol. 266, 167-173. doi: 10.1016/j.ijcard. 2018.01.062

Di Napoli, P., Taccardi, A. A., Grilli, A., Felaco, M., Balbone, A., Angelucci, D., et al. (2003). Left ventricular wall stress as a direct correlate of cardiomyocyte apoptosis in patients with severe dilated cardiomyopathy. Am. Heart J. 146, 1105-1111. doi: 10.1016/s0002-8703(03)00445-9

Efthimiadis, G. K., Pagourelias, E. D., Parcharidou, D., Gossios, T., Kamperidis, V., Theofilogiannakos, E. K., et al. (2013). Clinical characteristics and natural history of hypertrophic cardiomyopathy with midventricular obstruction. Circ. J. 77, 2366-2374. doi: 10.1253/circj.cj-12-1561

Elliott, P. M., Anastasakis, A., Borger, M. A., Borggrefe, M., Cecchi, F., Charron, P., et al. (2014). 2014 ESC guidelines on diagnosis and management of hypertrophic cardiomyopathy: the task force for the diagnosis and management of hypertrophic cardiomyopathy of the european society of cardiology (ESC). Eur. Heart J. 35, 2733-2779. doi: 10.1093/eurheartj/ehu284

Falicov, R. E., Resnekov, L., Bharati, S., and Lev, M. (1976). Mid-ventricular obstruction: a variant of obstructive cardiomyopathy. Am. J. Cardiol. 37, 432437. doi: 10.1016/0002-9149(76)90295-2

Friedman, B. M., and Dunn, M. I. (1995). Postinfarction ventricular aneurysms. Clin. Cardiol. 18, 505-511. doi: 10.1002/clc.4960180905

Genet, M., Lee, L. C., Nguyen, R., Haraldsson, H., Acevedo-Bolton, G., Zhang, Z., et al. (2014). Distribution of normal human left ventricular myofiber stress at end diastole and end systole: a target for in silico design of heart failure treatments. J. Appl. Physiol. 117, 142-152. doi: 10.1152/japplphysiol.00255.2014

Grossman, W. (1980). Cardiac hypertrophy: useful adaptation or pathologic process? Am. J. Med. 69, 576-584. doi: 10.1016/0002-9343(80)90471-4

Harada, K., Shimizu, T., Sugishita, Y., Yao, A., Suzuki, J., Takenaka, K., et al. (2001). Hypertrophic cardiomyopathy with midventricular obstruction and apical aneurysm: a case report. Jpn. Circ. J. 65, 915-919. doi: 10.1253/jcj.65.915

\section{DATA AVAILABILITY STATEMENT}

The original contributions presented in the study are included in the article/supplementary material, further inquiries can be directed to the corresponding author/s.

\section{AUTHOR CONTRIBUTIONS}

LD and XH designed the study. HZ, XH, and CY constructed the models and performed the numerical simulation. $\mathrm{HZ}$ and $\mathrm{XH}$ performed the statistical analysis of the data. $\mathrm{LD}, \mathrm{AL}$, and $\mathrm{XH}$ wrote the manuscript. All authors contributed to the article and approved the submitted version.

\section{FUNDING}

The research was funded in part by the National Natural Sciences Foundation of China (Grants 82000472 and 11671335) and the Fundamental Research Funds for the Central Universities (Grant 20720180004).

Huang, X., Deng, L., Zuo, H., Yang, C., Song, Y., Lesperance, M., et al. (2021). Comparisons of simulation results between passive and active fluid structure interaction models for left ventricle in hypertrophic obstructive cardiomyopathy. Biomed. Eng. Online 20:9.

Humphrey, J. D. (2002). Cardiovascular Solid Mechanics. New York, NY: SpringerVerlag.

Minami, Y., Kajimoto, K., Terajima, Y., Yashiro, B., Okayama, D., Haruki, S., et al. (2011). Clinical implications of midventricular obstruction in patients with hypertrophic cardiomyopathy. J. Am. Coll. Cardiol. 57, 2346-2355. doi: 10.1016/j.jacc.2011.02.033

Osawa, H., Fujimatsu, T., Takai, F., and Suzuki, H. (2011). Hypertrophic cardiomyopathy with apical aneurysm: left ventricular reconstruction and cryoablation for ventricular tachycardia. Gen. Thorac. Cardiovasc. Surg. 59, 354-358. doi: 10.1007/s11748-010-0695-7

Sacks, M. S., and Chuong, C. J. (1993). Biaxial mechanical properties of passive right ventricular free wall myocardium. J. Biomech. Eng. 115, 202-205. doi: $10.1115 / 1.2894122$

Semsarian, C., Ingles, J., Maron, M. S., and Maron, B. J. (2015). New perspectives on the prevalence of hypertrophic cardiomyopathy. J. Am. Coll. Cardiol. 65, 1249-1254. doi: 10.1016/j.jacc.2015.01.019

Tang, D., Yang, C., Del Nido, P. J., Zuo, H., Rathod, R. H., Huang, X., et al. (2016). Mechanical stress is associated with right ventricular response to pulmonary valve replacement in patients with repaired tetralogy of Fallot. J. Thorac. Cardiovasc. Surg. 151, 687-694. doi: 10.1016/j.jtcvs.2015.09.106

Tang, D., Yang, C., Geva, T., and Del Nido, P. J. (2010). Image-based patientspecific ventricle models with fluid-structure interaction for cardiac function assessment and surgical design optimization. Prog. Pediatr. Cardiol. 30, 51-62. doi: 10.1016/j.ppedcard.2010.09.007

Tang, D., Yang, C., Geva, T., Gaudette, G., and Del Nido, P. J. (2011). Multiphysics MRI-based two-layer fluid-structure interaction anisotropic models of human right and left ventricles with different patch materials: cardiac function assessment and mechanical stress analysis. Comput. Struct. 89, 1059-1068. doi: 10.1016/j.compstruc.2010.12.012

Türkoð̋lu, C., Gür, M., S̨eker, T., Selek, S̨, and Koçyiðit, A. (2016). The predictive value of M30 and oxidative stress for left ventricular remodeling in patients with anterior ST-segment elevation myocardial infarction treated with primary percutaneous coronary intervention. Coron. Artery Dis. 27, 690-695. doi: 10. 1097/mca.0000000000000416 
Walker, J. C., Ratcliffe, M. B., Zhang, P., Wallace, A. W., Fata, B., Hsu, E. W., et al. (2005). MRI-based finite-element analysis of left ventricular aneurysm. Am. J. Physiol. Heart Circ. Physiol. 289, H692-H700.

Yang, C., Tang, D., Haber, I., Geva, T., and Nido, P. J. D. (2007). In vivo MRIbased 3D FSI RV/LV models for human right ventricle and patch design for potential computer-aided surgery optimization. Comput. Struct. 85, 988-997. doi: 10.1016/j.compstruc.2006.11.008

Yin, Z., Zhang, W., Zhao, D., Sulejmani, F., Feng, Y., Huo, Y., et al. (2020). Cardiac wall mechanics analysis in hypertension-induced heart failure rats with preserved ejection fraction. J. Biomech. 98:109428. doi: 10.1016/j.jbiomech. 2019.109428

You, J., Wu, J., Zhang, Q., Ye, Y., Wang, S., Huang, J., et al. (2018). Differential cardiac hypertrophy and signaling pathways in pressure versus volume overload. Am. J. Physiol. Heart Circ. Physiol. 314, H552-H562.

Yu, H., Tang, D., Geva, T., Yang, C., Wu, Z., Rathod, R. H., et al. (2019). Ventricle stress/strain comparisons between tetralogy of Fallot patients and healthy using models with different zero-load diastole and systole morphologies. PLoS One 14:e0220328. doi: 10.1371/journal.pone.0220328

Conflict of Interest: CY was employed by company China United Network Communications Co., Ltd.

The remaining authors declare that the research was conducted in the absence of any commercial or financial relationships that could be construed as a potential conflict of interest.

Copyright (C) 2021 Deng, Zuo, Li, Yang and Huang. This is an open-access article distributed under the terms of the Creative Commons Attribution License (CC BY). The use, distribution or reproduction in other forums is permitted, provided the original author(s) and the copyright owner(s) are credited and that the original publication in this journal is cited, in accordance with accepted academic practice. No use, distribution or reproduction is permitted which does not comply with these terms. 